\title{
Evaluation of neutrophil-to-lymphocyte ratio prior to prostate biopsy to predict biopsy histology: Results of 1836 patients
}

\author{
Mehmet Ilker Gokce, MD; ${ }^{*}$ Nurullah Hamidi, MD; ${ }^{*}$ Evren Suer, MD; ${ }^{*}$ Semih Tangal, MD; ${ }^{\dagger}$ Adil Huseynov, MD; \\ Arif Ibiş, $M D^{*}$
}

*Department of Urology, Ankara University School of Medicine, Ankara, Turkey; 'Department of Urology, Ufuk University School of Medicine, Ankara, Turkey

Cite as: Can Urol Assoc J 2015;9(11-12):E761-5. http://dx.doi.org/10.5489/cuaj.3091 Published online November 4, 2015.

\section{Abstract}

Introduction: We evaluate the role of NLR prior to prostate biopsy to predict biopsy histology and Gleason score in patients with prostate cancer.

Methods: In this retrospective study, we evaluated data of patients underwent prostate biopsy between May 2005 and March 2015. We collected the following data: age, prostate-specific antigen (PSA), biopsy histology, Gleason score (GS) in prostate cancer patients, neutrophil counts, and lymphocyte counts. Patients were grouped as benign prostatic hyperplasia (BPH), prostate cancer, and prostatitis. The Chi square test was used to compare categorical variables and analysis of variance (ANOVA) was applied for continuous variables.

Results: Data of 1836 patients were investigated. The mean age, total PSA and neutrophil-lymphocyte ratio (NLR) of the population were $66.8 \pm 8.17$ years, $9.38 \pm 4.7 \mathrm{ng} / \mathrm{dL}$, and $3.11 \pm 1.71$, respectively. Patients were divided as follows: 625 in the group with BPH history, 600 in the prostatitis group, and 611 in the prostate cancer histology group. The mean NLR of the prostatitis group was higher compared to the prostate cancer and BPH groups $(p=0.0001)$. The mean NLR of the prostate cancer group was significantly higher compared to the BPH group $(p=0.002)$. The GS 8-10 group had a significantly higher mean NLR compared to GS 5-6 (3.64 vs. 2.54, $p=0.0001)$ and GS 7 (3.64 vs. 2.58, $p=0.0001)$ patients.

Conclusions: NLR was found to differ with regard to histology of prostate biopsy and higher GS was associated with higher NLR in patients with prostate cancer. However prostatitis prevents the use of NLR in predicting prostate cancer before a prostate biopsy. Also, the retrospective nature and lack of multivariate analysis in this study somewhat limits the relevance of these results.

\section{Introduction}

In Europe, prostate cancer is the most common cancer in males $>70$ years of age and is the second most common cause of cancer death. ${ }^{1}$ Its clinical relevance rises especially in the developed countries, and especially in elderly men. ${ }^{2}$

Biopsy is required for the histological diagnosis of prostate cancer and transrectal ultrasound (TRUS)-guided prostate biopsy is currently the standard route for gaining prostate tissue for histological examination. However, benign prostate hyperplasia (BPH) and prostatitis are the two commonly observed conditions resulting in prostate-specific antigen (PSA) elevation leading to a prostate biopsy. As TRUS-guided prostate biopsy is associated with significant complications, preventing unnecessary biopsies becomes very important. ${ }^{3}$ TRUS-guided prostate biopsies also raise the diagnostic rate of clinically insignificant prostate cancer. Various molecular biomarkers or imaging modalities have been suggested to increase the diagnostic accuracy of prostate biopsy, yet none of these methods are available for widespread use either due to lack of standardization and regional availability, or high cost.

The role of systemic inflammation in carcinogenesis and cancer progression has been studied recently and inflammatory markers have been associated with more aggressive disease in various types of cancer, including prostate cancer. $^{4-7}$ Neutrophil-to-lymphocyte ratio (NLR) has been studied as an inflammatory marker and neutrophilia and lymphocytopenia have been associated with poor survival outcomes. ${ }^{8,9}$ In prostate cancer patients receiving radiation therapy, higher NLR was associated with poor survival. ${ }^{6}$ In another study, patients with elevated NLR, receiving docetaxel-based chemotherapy had worse survival. ${ }^{7}$

To our knowledge, the role NLR prior to prostate biopsy to predict histological outcomes has not been investigated in any study. In this study, we identified the role of NLR prior to prostate biopsy to predict biopsy histology, and we evaluate the relationship of NLR with Gleason score (GS) in patients with prostate cancer. 
Gokce et al.

\section{Methods}

We retrospectively analyzed the data of 1836 Turkish patients who underwent TRUS-guided 12-core prostate biopsy between May 2005 and March 2015. Patient charts were reviewed and data regarding age, PSA level, biopsy histology, GS in prostate cancer patients, neutrophil counts, and lymphocyte counts were collected. NLR was calculated by dividing the neutrophil count by the lymphocyte count.

TRUS examinations and biopsies were performed using an ultrasound scanner (Toshiba SSA 250- A, Japan) equipped with a biplanar (6-MHz end-fire sector, $7-\mathrm{MHz}$ linear) transrectal probe, by transrectal route on axial plane. All patients underwent an equal number of biopsies (systematic 12-core biopsy) using an automatic spring-loaded device, with an 18-G needle (Promag, Mannan, FL). Systematic sextant and lateral biopsies from the base, middle and apical regions of the right and left peripheral zones were taken during the 12-core biopsy procedure.

Patients with a history of autoimmune or inflammatory diseases that may modify NLR levels, patients with a clinical suspicion or laboratory signs of bacterial or viral infection at the time of biopsy, patients with a history of chemotherapy, radiation therapy at any time were excluded from the study. Patients with a history of regular use of anti-inflammatory drugs or systemic steroids were also excluded from the study. Additionally, patients with symptomatic prostatitis, histology of high-grade prostatic intraepithelial neoplasia (PIN) or atypical small acinar proliferation (ASAP) were excluded due to the low number of patients.

Patients were grouped with regard to histology of the biopsy (BPH, prostate cancer and prostatitis) and were compared for age, PSA level, free/total PSA ratio, neutrophil count, lymphocyte count and NLR. To group patients with mixed histologies, patients with a benign prostate tissue, other than prostatitis or $\mathrm{BPH}$, were grouped in the $\mathrm{BPH}$ group. If the patient had both prostatitis and any other benign condition, he was grouped in the prostatitis group.

Statistical analysis was performed with SPSS ver. 20.0. Normal distribution of the data was investigated by the Kolmogorov-Simirnov test and data were expressed as the mean \pm standard deviation. The Chi square test was used to compare categorical variables and Student t-test and analysis of variance (ANOVA) was applied for continuous variables of the groups based on biopsy histology. The Bonferroni test was applied to determine intergroup differences.

\section{Results}

During the study period, prostate biopsy was performed in 2144 patients; of these, 308 did not meet the inclusion criteria for lack of data regarding blood counts or the presence of high-grade and ASAP in the pathology report. High-grade PIN and ASAP were detected in 30 and 22 patients, respectively. In total, the data of 1836 patients were investigated and the mean age of the population was $66.8 \pm 8.17$ years. The mean total PSA level and free/total PSA ratio were $9.38 \pm 4.7 \mathrm{ng} / \mathrm{dL}$ (range: $0.9-568 \mathrm{ng} / \mathrm{mL}$ ) and $0.225 \pm 0.188$, respectively. The mean neutrophil count, lymphocyte count and NLR were $5.39 \pm 2.31,2.06 \pm 0.77$ and $3.11 \pm 1.71$, respectively.

Regarding the pathology reports, there were 625, 600 and 611 patients with $\mathrm{BPH}$, prostatitis, and prostate cancer, respectively. When the 3 groups of histology were compared, we found that mean age and total PSA levels in the prostate cancer group were significantly higher compared to the other 2 groups; moreover, the free/total PSA ratio of the prostate cancer group was significantly lower compared to the other 2 groups (Table 1 ).

The mean neutrophil count of the prostatitis group was significantly higher, and the mean lymphocyte count of the prostatitis group was significantly lower compared to the prostate cancer and BPH groups. Therefore, the mean NLR of the prostatitis group was significantly higher compared to the prostate cancer and $\mathrm{BPH}$ groups. The mean neutrophil count and NLR of the prostate cancer group was significantly higher compared to the BPH group, but the difference of the mean lymphocyte counts was not significantly different (Table 1). The $p$ values of the 3 histology groups were compared using the Bonferroni analysis (Table 2).

The patients in the prostate cancer group were divided according to GS (Table 3). When compared for NLR, the GS 8-10 group had a significantly higher mean NLR compared to the GS 5-6 and GS 7 groups. The differences in the GS 5-6 and GS 7 patients were not statistically significant $(p=0.955)($ Table 3$)$.

\begin{tabular}{|c|c|c|c|c|}
\hline & $\mathrm{BPH}(\mathrm{n}=625)$ & Prostatitis $(n=600)$ & Prostate cancer $(n=611)$ & $p$ value \\
\hline Age, years (mean $\pm S D$ ) & $65.6 \pm 7.6$ & $66.1 \pm 7.7$ & $68.8 \pm 8.8$ & 0.0001 \\
\hline Total PSA, ng/mL (mean \pm SD) & $7.05 \pm 3.4$ & $8.23 \pm 4.4$ & $12.9 \pm 5.1$ & 0.0001 \\
\hline Free/total PSA ratio (\%) & $0.25 \pm 0.13$ & $0.23 \pm 0.14$ & $0.19 \pm 0.12$ & 0.0001 \\
\hline Neutrophil count, $\times 103$ & $4.54 \pm 1.7$ & $6.65 \pm 2.2$ & $5.02 \pm 3.3$ & 0.0001 \\
\hline Lymphocyte count, $\times 103$ & $2.19 \pm 0.67$ & $1.83 \pm 0.61$ & $2.15 \pm 0.94$ & 0.0001 \\
\hline NLR (\%) & $2.39 \pm 2.1$ & $4.07 \pm 3.01$ & $2.91 \pm 2.67$ & 0.0001 \\
\hline
\end{tabular}

BPH: benign prostatic hyperplasia; SD: standard deviation; PSA: prostate-specific antigen; NLR: neutrophil-to-lymphocyte ratio. 


\begin{tabular}{|c|c|c|c|c|}
\hline & & $\mathrm{BPH}(\mathrm{n}=625)$ & Prostatitis $(n=600)$ & Prostate cancer $(n=611)$ \\
\hline & $\mathrm{BPH}$ & 1 & 0.763 & 0.0001 \\
\hline \multirow[t]{3}{*}{ Age, years } & Prostatitis & 0.763 & 1 & 0.0001 \\
\hline & Prostate cancer & 0.0001 & 0.0001 & 1 \\
\hline & BPH & 1 & 0.988 & 0.0001 \\
\hline \multirow[t]{3}{*}{ Total PSA, ng/mL } & Prostatitis & 0.998 & 1 & 0.0001 \\
\hline & Prostate cancer & 0.0001 & 0.0001 & 1 \\
\hline & $\mathrm{BPH}$ & 1 & 0.05 & 0.0001 \\
\hline \multirow[t]{3}{*}{ Free/total PSA ratio (\%) } & Prostatitis & 0.05 & 1 & 0.0001 \\
\hline & Prostate cancer & 0.0001 & 0.0001 & 1 \\
\hline & $\mathrm{BPH}$ & 1 & 0.0001 & 0.0001 \\
\hline \multirow[t]{3}{*}{ Neutrophil count } & Prostatitis & 0.0001 & 1 & 0.0001 \\
\hline & Prostate cancer & 0.0001 & 0.0001 & 1 \\
\hline & $\mathrm{BPH}$ & 1 & 0.0001 & 0.889 \\
\hline \multirow[t]{3}{*}{ Lymphocyte count } & Prostatitis & 0.0001 & 1 & 0.0001 \\
\hline & Prostate cancer & 0.889 & 0.0001 & 1 \\
\hline & $\mathrm{BPH}$ & 1 & 0.0001 & 0.002 \\
\hline \multirow[t]{2}{*}{ NLR (\%) } & Prostatitis & 0.0001 & 1 & 0.0001 \\
\hline & Prostate cancer & 0.002 & 0.0001 & 1 \\
\hline
\end{tabular}

BPH: benign prostatic hyperplasia; PSA: prostate-specific antigen; NLR: neutrophil-to-lymphocyte ratio.

\section{Discussion}

TRUS-guided prostate biopsy is essential in diagnosing prostate cancer in most cases. The most common indication to perform prostate biopsy is elevated PSA levels. However, benign conditions, such as BPH, acute or chronic prostatitis, or trauma, can also elevate PSA levels, decrease the specificity of PSA, and lead to unnecessary biopsies. Also up to $20 \%$ of men with prostate cancer may be misdiagnosed in the first prostate biopsy. ${ }^{10}$ Another important point regarding prostate biopsies is the diagnosis of clinically insignificant prostate cancer with GS 6 and limited volume within the prostate.

To overcome these problems, several molecular, biochemical markers have been studied. Additionally, there is growing interest on multiparametric magnetic resonance imaging (MRI) studies to increase the diagnostic accuracy of the prostate biopsy. Unfortunately, neither molecular nor biochemical markers are universally accepted due to lack of availability or cost. There is a need to find biomarker that is cheap, widely available and applicable to increase the

\begin{tabular}{lc}
\hline $\begin{array}{l}\text { Table 3. Gleason score grouping of patients in the prostate } \\
\text { cancer group }\end{array}$ \\
\hline Gleason score & No. patients \\
\hline 5 & 30 \\
6 & 193 \\
7 & 194 \\
8 & 83 \\
9 & 92 \\
10 & 19 \\
\hline
\end{tabular}

diagnostic accuracy of prostate biopsy and prevent unnecessary biopsies.

The role of systemic inflammation in carcinogenesis has been studied in various cancer types. ${ }^{10-13}$ Also markers of systemic inflammation, such as $\mathrm{C}$ - reactive protein (CRP), neutrophil count, platelet count and NLR, have been investigated in various cancer types. ${ }^{14-17}$ However, the effect of systemic inflammation on PSA levels and related results of prostate biopsies have not been studied previously. McDonald and colleagues studied the relationship of inflammatory markers and PSA levels and found that serum CRP, fibrinogen and NLR levels were higher in men with elevated PSA levels compared to men with normal PSA levels. However in this study, the results of prostate biopsies were not investigated. ${ }^{18}$

In our study we evaluated the role of NLR prior to prostate biopsy to predict the biopsy histology and to assess its association with GS in patients with prostate cancer. We found that men with prostate cancer have higher NLR compared to men with BPH histology, but lower NLR compared to men with prostatitis histology. The same relationship was also observed for the neutrophil counts, but not for the lymphocyte counts. There was no significant difference between the prostate cancer and $\mathrm{BPH}$ groups regarding lymphocyte counts. NLR was not effective in discriminating benign $(\mathrm{BPH}$,

\begin{tabular}{lcccc}
\hline \multicolumn{5}{c}{ Table 4. Comparison of the NLR of GS subgroups } \\
\hline & $\begin{array}{c}\text { GS 5-6 } \\
\text { (n = 223) }\end{array}$ & $\begin{array}{c}\text { GS 7 } \\
\text { (n= 194) }\end{array}$ & $\begin{array}{c}\text { GS 8-10 } \\
\text { (n = 194) }\end{array}$ & p value \\
\hline NLR $(\%)$ & 2.54 & 2.58 & 3.64 & 0.001 \\
\hline
\end{tabular}

NLR: neutrophil-to-lymphocyte ratio; GS: Gleason score. 
prostatitis) histology and prostate cancer; the mean NLR of the prostate cancer group was located between the two benign histologies.

The role of neutrophil counts, but not the NLR, was previously studied by Fujita and colleagues. ${ }^{19}$ They evaluated results of 323 patients who underwent prostate biopsy, of whom 203 were positive for prostate cancer. They also compared patients with prostate cancer and benign histology. Their results revealed a significantly higher neutrophil count in patients with prostate cancer and it was found to be an independent prognostic marker in the multivariate analysis. However the authors did not classify the benign histology subtypes and concluded that elevated neutrophil count may be a good indicator of a benign prostate biopsy. They also suggested that men with a low neutrophil count and an increase of serum PSA should strongly be considered for biopsy. ${ }^{19}$ In our study we also found that neutrophil count of our prostate cancer patients was higher than in those with benign histology other than prostatitis. However, the neutrophil count in the prostatitis group was higher than in the prostate cancer and $\mathrm{BPH}$ groups. It is worth noting that the neutrophil count may vary even within the benign histologies.

An important result from our study is the correlation of higher GS with NLR. GS 8-10 patients, with a worse prognosis compared to men with GS $\leq 7$, had significantly higher mean NLR levels (GS 5-6: 3.64 vs. 2.54, $p=0.0001$ and for GS 7: 3.64 vs. $2.58, p=0.0001$ ). This is the first study to evaluate the association of GS and NLR levels. Previous studies evaluated the role of NLR in predicting outcome in castration-resistant prostate cancer patients receiving docetaxel, or patients receiving radiation therapy for localized disease. However, the role of NLR to predict treatment outcomes after radical prostatectomy has also not been studied.

\section{Limitations of the study}

The most important drawback of our study is its retrospective nature. However, we believe that the number of patients in the study mitigates this disadvantage; moreover, patient follow-up was not a core part of our study. Another important point is the low number of patients with ASAP and high-grade PIN. We excluded these cases due to their low number; this exclusion led to potential heterogeneity bias within the study population. Also we could not perform receiver operating characteristic analysis to find the threshold value of NLR to determine prostate cancer, due to the mean NLR values of prostate cancer group being between the two benign histologies.

We could also not verify other markers of systemic inflammation, such as CRP or procalcitonin, due to the retrospective nature of the study. Our patients are asymptomatic, therefore serum CRP or procalcitonin levels were not studied in most of patients. Due to the retrospective nature of the study, we could not evaluate the role of other confounders, such as smoking, body mass index, and presence of metabolic syndrome, which would have been associated with the inflammatory response and may be involved in a multivariate analysis.

\section{Conclusion}

NLR prior to prostate biopsy was higher in patients with prostatitis compared to prostate cancer or $\mathrm{BPH}$. Also prostate cancer patients had higher mean NLR compared to BPH patients. Higher GS was also associated with higher NLR in prostate cancer patients. The results support the role of inflammation in the development of prostate cancer; however prostatitis, a common inflammatory condition of prostate gland, prevents the use of NLR in predicting prostate cancer prior to prostate biopsy.

Competing interests: The authors declare no competing financial or personal interests.

This paper has been peer-reviewed.

\section{References}

1. Boyle P, Ferlay J. Cancer incidence and mortality in Europe 2004. Ann Oncol 2005;16:481-8. http:// dx.doi.org/10.1093/annonc/mdi098

2. Arnold M, Karim-Kos HE, Coebergh JW, et al. Recent trends in incidence of five common cancers in 26 European countries since 1988: Analysis of the European Cancer Observatory. Eur J Cancer 2015;51:116487. http://dx.doi.org/10.1016/i.ejca.2013.09.002

3. Rosario DJ, Lane JA, Metcalfe C, et al. Short term outcomes of prostate biopsy in men tested for cancer by PSA: A prospective evaluation within the ProtecT study. BMJ 2012;344:d7894. http://dx.doi. org/10.1136/bmi.d7894

4. Casamassima $A$, Picciariello $M$, Quaranta $M$, et al. C-reactive protein: A biomarker of survival in patients with metastatic renal cell carcinoma treated with subcutaneous interleukin-2 based immunotherapy. J Urol 2005;173:52-5. http://dx.doi.org/10.1097/01.ju.0000146713.50673.e5

5. Ohno $Y$, Nakashima J, Ohori $M$, et al. Pretreatment neutrophil-to-lymphocyte ratio as an independent predictor of recurrence in patients with nonmetastatic renal cell carcinoma. J Urol 2010;184:873-8. http://dx.doi.org/10.1016/i.juro.2010.05.028

6. Langsenlehner T, Thurner EM, Krenn-Pilko $S$, et al. Validation of the neutrophil-to-lymphocyte ratio as a prognostic factor in a cohort of European prostate cancer patients. World I Urol 2015;1-7; Epub 24 January 2015; http://dx.doi.org/10.1007/s00345-015-1494-7

7. Nuhn P, Vaghasia AM, Goyal J, et al. Association of pretreatment neutrophil-to-lymphocyte ratio (NLR) and overall survival $(O S)$ in patients with metastatic castration-resistant prostate cancer (mCRPC) treated with first-line docetaxel. BJU Int 2014;114:E1 1-7. http://dx.doi.org/10.1111/bju.12531

8. Atzpodien J, Royston $P$, Wandert $T$, et al. Metastatic renal carcinoma comprehensive prognostic system. Br J Cancer 2003;88:348-53. http://dx.doi.org/10.1038/si.bic.6600768

9. Fumagalli LA, Vinke J, Hoff W, et al. Lymphocyte counts independently predict overall survival in advanced cancer patients: A biomarker for IL-2 immunotherapy. J Immunother 2003;26:394-402. http://dx.doi. org/10.1097/00002371-200309000-00002

10. De Marzo AM, Platz EA, Sutcliffe $S$, et al. Inflammation in prostate carcinogenesis. Nat Rev Cancer 2007;7:256-69. http://dx.doi.org/10.1038/nrc2090

11. Coussens LM, Werb Z. Inflammation and cancer. Nature 2002;420:860-7. http://dx.doi.org/10.1038/ nature01322

12. Heikkila K, Ebrahim S, Lawlor DA. A systematic review of the association between circulating concentrations of C reactive protein and cancer. J Epidemiol Community Health 2007;61:824-33. http://dx.doi. org/10.1136/jech.2006.051292 
13. dos Santos Silva I, De Stavola BL, Pizzi C, et al. Circulating levels of coagulation and inflammation markers and cancer risks: Individual participant analysis of data from three long-term cohorts. Int I Epidemiol 2010;39:699-709. http://dx.doi.org/10.1093/iie/dyq012

14. Guthrie GJK, Charles KA, Roxburgh CSD, et al. The systemic inflammation-based neutrophil- lymphocyte ratio: Experience in patients with cancer. Crit Rev Oncol Hematol 2013;88:218-30. http://dx.doi. org/10.1016/i.critrevonc.2013.03.010

15. Roxburgh CSD, McMillan DC. Role of systemic inflammatory response in predicting survival in patients with primary operable cancer. Future Oncol 2009;6:149-63. http://dx.doi.org/10.2217/fon.09.136

16. Beer TM, Lalani AS, Lee S, et al. ASCENT Investigators. C-reactive protein as a prognostic marker for men with androgen-independent prostate cancer: Results from the ASCENT trial. Cancer 2008;112:2377-83. http://dx.doi.org/10.1002/cncr.23461

17. Hamidi N, Gökçe MI, Süer E, et al. Evaluation of increased preoperative serum high sensitive C-reactive protein and procalcitonin levels on grade and stage of clear cell renal cell carcinoma. Clin Nephrol 2015;83:225-30. http://dx.doi.org/10.5414/cn108448
18. MCDonald AC, Vira MA, Vidal AC, et al. Association between systemic inflammatory markers and serum prostate-specific antigen in men without prostatic disease - the 2001-2008 National Health and Nutrition Examination Survey. Prostate 2014;74:561-7. http://dx.doi.org/10.1002/pros.22782

19. Fujita $\mathrm{K}$, Imamura $\mathrm{R}$, Tanigawa $\mathrm{G}$, et al. Low serum neutrophil count predicts a positive prostate biopsy. Prostate Cancer Prostatic Dis 2012;15:386-90. http://dx.doi.org/10.1038/pcan.2012.27

Correspondence: Dr. Nurullah Hamidi, Department of Urology, Ankara University School of Medicine, Ankara, Turkey; dr.nhamidi86@gmail.com 